\title{
ERRATA
}

\section{A PARTIAL ORDER ON THE SET OF PRIME KNOTS WITH UP TO 11 CROSSINGS}

[Journal of Knot Theory and Its Ramifications, Vol. 20, No. 2 (2011) 275-303]

\author{
KEIICHI HORIE \\ DeNA Co., Ltd, Japan \\ horie.keiichi@dena.jp \\ TERUAKI KITANO \\ Department of Information Systems Science, \\ Faculty of Engineering, Soka University, \\ 1-236 Tangi-cho, Hachioji-shi, Tokyo, 192-8577, Japan \\ kitano@soka.ac.jp \\ MINEKO MATSUMOTO \\ Division of Information Systems Science, \\ Graduate School of Engineering, Soka University, \\ 1-236 Tangi-cho, Hachioji-shi, \\ Tokyo, 192-8577, Japan

\section{MASAAKI SUZUKI} \\ Department of Mathematics, Akita University, \\ 1-1 TegataGakuenmachi, Akita 010-8502, Japan \\ macky@math.akita-u.ac.jp
}

Received 8 July 2011

Accepted 9 July 2011

Published 8 February 2012

\begin{abstract}
Keywords: Prime knot; surjective homomorphism; partial order; twisted Alexander polynomial.

Mathematics Subject Classification 2010: 57M25, 57M05, 57M27
\end{abstract}

The word "meridian-preserving" is missing in several sentences. We should replace all the phrases "surjective homomorphism" with "meridian-preserving surjective homomorphism" in this paper. Furthermore, the Wirtinger presentations should be chosen as the presentations of knot groups in Proposition 2.4. 
Proposition 2.4 should say:

We take the Wirtinger presentations of knot groups $G\left(K_{1}\right), G\left(K_{2}\right)$. Assume that there exists a meridian-preserving surjective homomorphism $\varphi: G\left(K_{1}\right) \rightarrow G\left(K_{2}\right)$. Then for any representation $\rho_{2}: G\left(K_{2}\right) \rightarrow S L\left(2 ; \mathbb{F}_{p}\right)$ and $\rho_{1}=\rho_{2} \circ \varphi, \Delta_{K_{1}, \rho_{1}}(t)$ is divisible by $\Delta_{K_{2}, \rho_{2}}(t)$. More precisely, the denominator of $\Delta_{K_{1}, \rho_{1}}(t)$ is same as the denominator of $\Delta_{K_{2}, \rho_{2}}(t)$ and the numerator of $\Delta_{K_{1}, \rho_{1}}(t)$ can be divided by the numerator of $\Delta_{K_{2}, \rho_{2}}(t)$. 